\title{
TAM KONTÜR ZİRKONYA RESTORASYONLARIN FARKLI ALL ON FOUR

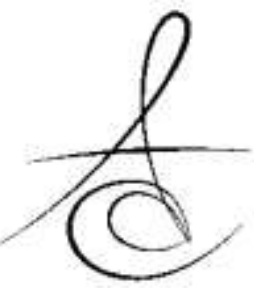 MANDİBULAR İMPLANT MODELLERİNDE BİYOMEKANİK DAVRANIŞLARI: 3 BOYUTLU SONLU ELEMANLAR STRES ANALİİ ÇALIŞMASI \\ BIOMECHANICAL BEHAVIORS OF FULL COUNTOR ZIRCONIA RESTORATIONS ON DIFFERENT ALL ON FOUR MANDIBULAR IMPLANT MODELS: A 3 DIMENSIONAL FINITE ELEMENT STRESS ANALYSIS STUDY
}

\author{
Dr. Öğr. Üy. Gonca DESTE*
}

\author{
Doç. Dr. Rukiye DURKAN**
}

\author{
Makale Kodu/Article code: 3943 \\ Makale Gönderilme tarihi; 26.12 .2018 \\ Kabul Tarihi: 16.04.2019
}

DOI : $10.17567 /$ ataunidfd. 554400

Gonca Deste: ORCID ID: 0000-0002-5481-0063

Rukiye Durkan: ORCID ID: 0000-0002-3381-4073

\section{ÖZ}

Amaç: Aşıı rezorbe mandibular tam dişsizlik durumunda implant uygulamalarında ve oklüzyonun sağlanmasında zorluklar ve hareketli protez kullanımı yaygın gözlenen problemlerdir. Mandibular 4 implant üzerine yapılan sabit tam ark protetik restorasyonlar alternatif bir metod sağlamaktadır. İmplant sayısının az olması, cerrahi işlemlerin kısa sürmesi ve sabit protez avantajı bu yöntemin özellikleridir. Bu çalışmanın amacı estetik ve son teknoloji ürünü olan monolitik zirkonya CAD-CAM seramik materyallerin farklı mandibular all on four implant tasarımlarında uygulanmasının biyomekanik özellikler bakımından değerlendirilmesidir.

Materyal ve metod: Katana CAD-CAM monolitik zirkonya tam ark sabit restorasyon, Biomed implant ve abutment materyalleri kullanıldı. 4 adet mandibular all on four implant tasarımlı model hazırlandı. 1. model; anterior implantlar vertikal ve posterior implantlar $15^{0}$ açll, kantilever $5 \mathrm{~mm},(Z-15,5)$; 2. model; kantilever $9 \mathrm{~mm}(Z-15,9)$; 3. model anterior implantlar vertikal posterior implantlar $30^{\circ}$ açıll, kantilever $5 \mathrm{~mm}(Z-30,5)$; 4.model; kantilever $9 \mathrm{~mm}(Z-30,9)$. Yükleme koşulu; 1. molar yüklemesi sağ kantilever uzantısının merkezinden oklüzyon düzlemine $30^{\circ}$ açılı ve $150 \mathrm{~N}$ kuvvet uygulandı. Üç boyutlu sonlu elemanlar stres analiz yöntemi ile mandibular kortikal ve trabeküler kemik, implant ve protetik restorasyonlardaki biyomekanik stres dağııımı ölçüldü. Statik lineer analiz yapıldı.

Bulgular: Bütün parametreler birlikte değerlendirildiği zaman CAD-CAM seramik materyal, kantilever uzunluğu ve stres dağılımının tipleri arasında önemli bağlantılar olduğu tespit edildi. Kantilever uzunluğu 5 mm'den 9 mm'ye çıktığı zaman kortikal ve trabeküler kemikte maksimum ve minimum asal gerilme stres değerleri yükseldi. Posterior implant açılanması $15^{\circ}$ 'den $30^{\circ \prime}$ ye çıtığı zaman implantlarda von Mises değerleri azaldı.

Sonuç: Monolitik zirkonya restorasyon $30^{\circ}$ posterior implant açılanması ve $5 \mathrm{~mm}$ kantilever uzantısı biyomekanik olarak kullanilabilir. Mandibular all on four implant destekli tam ark sabit estetik CAD-CAM restorasyonlarda biyomekanik ve klinik açıdan kantileverin kısaltılması implant açısının arttırılması ve rijit tek tabaka restorasyonların yapılması önerilebilir.

Anahtar kelimeler: monolitik zirkonya, üç boyutlu sonlu elemanlar stres analizi, all on four implant tasarımı

ABSTRACT

Aim: Difficulties in the implementation of implants and providing occlusion and the use of removable prostheses are common problems in case of extremely resorbed mandibular edentulism. The fixed full arch prosthetic restorations on the mandibular 4 implant provide an alternative method. The low number of implants, short duration of surgical procedures, and fixed prosthesis are the characteristics of this method. The aim of this study is to evaluate the application of aesthetic\& state of the art technology product of monolithic zirconia CAD-CAM ceramic materials in different mandibular "all four implant" designs in terms of biomechanical properties.

Material\&Method: Katana CAD-CAM monolithic zirconia full-arch fixed restoration, Biomed implant and abutment materials were used. 4 models of mandibular "all-on-four implants" were prepared. 1. model; vertical anterior implants and $15^{\circ}$ angled posterior implants, cantilever $5 \mathrm{~mm},(Z-15,5)$; 2 . model; cantilever $9 \mathrm{~mm}(Z-15,9), 3$. model; vertical anterior implants, 30 angled posterior implants, cantilever $5 \mathrm{~mm}(Z-30,5)$; 4. model; cantilever $9 \mathrm{~mm}(Z-30,9)$. Loading condition; The 1st molar loading was applied from the center of the right cantilever extension to the occlusion plane with an angle of 30 degrees and a force of $150 \mathrm{~N}$. Biomechanical stress distribution of mandibular cortical and trabecular bone, implant and prosthetic restorations were measured by three dimensional finite element stress analysis method. Static linear analysis was performed.

Results: When all parameters were evaluated together, it was found that there were significant connections between the CADCAM ceramic material, the length of the cantilever and types of stress distribution. When cantilever length was increased from 5 $\mathrm{mm}$ to $9 \mathrm{~mm}$, maximum and minimum principal stress values in cortical and trabecular bone were increased. When posterior implant angles increased from 15 to 30 degrees, the von Mises values of the implants decreased.

Conclusion: Monolithic zirconia restoration can be used biomechanically with 30 degree posterior implant angle and $5 \mathrm{~mm}$ cantilever extension. Biomechanically shortening cantilever, increasing implant angle and rigid monolithic restorations can be suggested on mandibular "all-on-four implant" supported full-arch fixed aesthetic CAD-CAM restorations.

Key words: monolithic zirconia, three dimensional finite element stress analysis, all on four implant design

*Bursa Uludağ Üniversitesi, Diş Hekimliği Fakültesi, Protetik Diş Tedavisi A.D.

** Afyonkarahisar Sağlık Bilimleri Üniversitesi, Diş Hekimliği Fakültesi Protetik Diş Tedavisi A.D, Afyonkarahisar

Kaynakça Bilgisi: Deste G, Durkan R. Tam Kontür Zirkonya Restorasyonların Farklı All On Four Mandibular İmplant Modellerinde Biyomekanik

Davranışları: 3 Boyutlu Sonlu Elemanlar Stres Analizi Çalışması. Atatürk Üniv Diş Hek Fak Derg 2020; 30: 424-33.

Citation Information: Deste G, Durkan R. Biomechanical Behaviors of Full Countor Zirconia Restorations on Different All On Four Mandibular Implant

Models: A 3 Dimensional Finite Element Stress Analysis Study. J Dent Fac Atatürk Uni 2020; 30: 424-33. 


\section{GİRIŞ}

Bilgisayar destekli tasarım ve bilgisayar destekli üretim (Computer-aided design and computer-aided manufacturing (CAD-CAM)) yöntemleri kullanılarak işlenen monolitik zirkonya seramik sistemleri yüksek dayanıklıık ve eğilme direncine sahip estetik tam seramik materyallerdir. ${ }^{1,2}$ Monolitik zirkonya seramik materyalle- rin konvansiyonel kor zirkonya seramiklere oranla pek çok avantajları bulunmaktadır. ${ }^{1}$ Alümina oranı \%0,1-0,05 düzeyine düşürülerek translusent özellik arttırıla- rak semitranslusent bir seramik olduğu rapor edilmektedir. ${ }^{2-4}$ Yaklaşık $40 \mathrm{~nm}$ nanopartiküler yapıda, flexural dayanıkılığı $1100 \mathrm{MPa}$ düzeyinde, elastik modülüsü 222 GPa değerindedir. ${ }^{5,6}$

Üreticilere göre, bu materyaller yüksek dirençli ve estetik olarak kabul edilmektedirler. Özellikle tam ark implant destekli sabit protetik restorasyonlarda seramik materyallerin yüksek elastik modülüne sahip, rijit ve estetik olması önemlidir. ${ }^{3-5}$ Tam ark implant üstü CAD-CAM monolitik zirkonya sabit dental köprü protezleri ile 5-10 yıl arası kısa dönemlerde klinik olarak estetik ve dayanıklılık bakımından oldukça başarılı sonuçlar elde edilebilmektedir. ${ }^{5}$

Rezorbe mandibular tam dişsizlik vakalarında; implant destekli sabit protezler veya overdenture protezler yapılabilmekte iken son yıllarda özellikle estetik ve tutuculuk özelliklerinden dolayı sabit tam ark restorasyonlar yapılmaktadır. ${ }^{3,4}$ Misch'e göre mandibular implant destekli protezlerde farklı implant sayı ve yerleşimleri ile parçalı tam ark sabit protezlerin yapılması önerilmektedir.7 Ancak mandibular rezorbe kretlerde posterior mandibulanın rezorpsiyonuna bağlı olarak mandibular kanalın limitasyonu posterior bölgede implant uygulamalarında zorluklar ile karşılaşılmaktadır. ${ }^{8,9}$ Buna çözüm olarak posterior kısa implantlar, geniş çaplı implantların uygulanması veya cerrahi olarak mandibular sinir repozisyonları, posterior kemik greft uygulamaları yapılmakla birlikte bu konuda yeterince iyi sonuçlar elde edilememiştir. ${ }^{8-10}$ Ayrıca hastalar ve hekimler için bu tür cerrahi uygulamalar travmatik, ağrılı ve uzun süreli iyileşme dönemleri gibi dezavantajları beraberinde getirdikleri ifade edilmektedir. ${ }^{11}$

Bu olumsuzluklara çözüm bulmak amacı ile 2003 yılında Malo ve ark tarafından tanımlanan all on four implant destekli protetik restorasyonların mandibuler tam dişsizlik vakalarında kullanımları sunulmuştur. ${ }^{9}$ All on four implant dizaynında mandibular lateral ve/veya kanin bölgesine iki implant vertikal ve oklüzyon düzlemine dik olarak ve posterior bölgede interfo- raminal alana 1 . ve/veya 2 . Premolar dişler bölgesine $15^{0}-45^{0}$ açılı ve oklüzyon düzlemine göre distale eğimli olarak yerleştirilen iki implant, toplam 4 implant (Nobel Biocare, Göteborg, Sweden) ile tam ark vida tutuculu sabit restorasyon yapılması önerilmiştir. ${ }^{9}$ Geçici protezlerin hemen yapılarak yüklenmesi (8-48 saat cerrahi sonrası) ve 3 aylık periyot sonrasında daimi sabit protezlerin yapılması şeklindedir. ${ }^{8-11}$ Mandibular restorasyonların uzun dönem klinik başarısında interforaminal anatomik bölgede bulunan kemik yoğunluğu oldukça önemlidir. 4 implantın yerleştirilmesi için yeterli kemik varlığı en az $5 \mathrm{~mm}$ kemik genişliği, mental foramenler arası en az 8-10 mm kemik yüksekliği, abutment, alt yapı ve protetik restorasyona yer sağlamak için arklar arası mesafenin en az 20 mm olması gerekmektedir. ${ }^{12,13}$

All on four mandibular tam ark implant destekli sabit restorasyonlarda protetik restorasyona, kortikal ve trabeküler kemiğe ve implantlara gelen oklüzal kuvvetlerin dengelenmesi ve bu yapılara zarar vermemesi gerekmektedir. ${ }^{13} \mathrm{Bu}$ kuvvetlerin ölçülmesinde üç boyutlu finite element analizleri (3D-FEA) kullanılmaktadır. 3D-FEA yönteminde materyal veya dokular gerçek boyutlarında matematiksel modele dönüştürülebilirler, analizler bilgisayar destekli yapıldığı için tekrar edilebilir niteliğe sahiptir. Deneysel yöntemlere göre daha hassas sonuçlar elde edilebilir ayrıca gerilme değerleri detaylı olarak ve kısa sürede elde edilebilir. ${ }^{14,15}$

3D-FEA sisteminde maksimum asal gerilim değerleri (maximum principal stresses)- Çekme gerilmesi Pozitif değerdir ve en yüksek gerilme stresini (tensile stress) ifade eder (Maks. PS veya $\sigma 1) .{ }^{14}$ Ara Asal Gerilme (Intermediate Principal Stress): Ara değerleri ifade eder ( $\sigma 2)$. Minimum asal gerilim değerleri (minimum principal stresses)-basma gerilmesi negatif değerdir ve en yüksek sıkışma stresini (compressive stress) ifade eder (Min. PS veya $\sigma 3$ ). 3D-FEA analiz sonuçlarında elde edilen verilerde pozitif değerler maksimum asal gerilme değerlerini, negatif değerler ise minimum asal gerilme değerlerini ifade etmektedir. ${ }^{14}$ Bir stres elemanında belirgin ölçüde hangi asal gerilme değeri daha büyük mutlak değere sahip ise, o stres elemanı daha büyük olan asal gerilme tipinin etkisi altındadır. 3D-FEA von Misses stres değerleri (equivalent stresses) ile tüm yapıda oluşan stres değerleri elde edilebilir. Von Mises stres değerleri ile arayüz bağlantılarında oluşan stresler nitelik ve nicelik yönünden değerlendirilir. ${ }^{16}$ Üç boyutlu stres analizinde streslerin yoğun olarak gözlendiği noktalardan stres 
değerleri tespit edilerek modelin sol tarafında bulunan skaladan stres miktarı MPa birimi olarak tespit edilir. 3D-FEA von Mises stres değerleri ve çekme stresleri kırmızıda en çok iken maviye doğru azalmaktadır. Basma stresleri için mavi renk en yüksek stres değerlerini göstermektedir. 3D-FEA basma stresleri negatif değerler ile gösterilmekte ve mutlak değeri kullanılmaktadır. ${ }^{16} \mathrm{Bu}$ yöntem biyomekanik stres dağılımının tespit edilmesinde önemli ölçüm sonuçları vermekle birlikte restorasyonların planlanmasında ve uzun dönem başarısında da yol gösterici olduğu rapor edilmektedir. ${ }^{16}$

3D-FEA yöntemi kullanılarak mandibular all on four implant destekli tam ark sabit protetik restorasyonlarda biyomekanik analizlere yönelik veri yetersizdir. ${ }^{17,18}$ Özellikle bu tür tasarımlarda protetik restorasyon materyali olarak CAD-CAM monolitik zirkonya kullanımlarına yönelik literatürde bir veriye rastlanmamıştır. Bu nedenle bu çalışmanın amacı estetik CAD-CAM materyalleri olan monolitik zirkonya tam ark sabit restorasyonların 4 farklı mandibular all on four tasarımlarına göre hazırlanmış olan dijital modeller üzerinde 3D-FEA yöntemi ile biomekanik analiz sonuçlarından von Mises stres değerleri, maksimum ve minimum asal gerilme değerlerinin elde edilmesidir. ${ }^{17,18}$

Bu çalışmada öngörülen 2 hipotez şunlardır: iki farklı kantilever uzunluğunun restorasyon, kemik veya implantlarda oluşturdukları stres değerleri benzerdir ve farklı posterior implant açılanmaları 3D-FEA biyomekanik olarak stres iletiminde önemli farklılık oluşturmamaktadır.

\section{MATERYAL ve METOD}

Çalışmada kullanılan monolitik zirkonya CADCAM seramik ve implant materyaller Tablo 1'de listelenmektedir. Materyallere ait elastik modülüs ve poison oranları Tablo 2'de listelenmektedir.

Tablo 1. Kullanılan materyaller

\begin{tabular}{|c|c|c|}
\hline Materyal & Kimyasal kompozisyonu & Üretici firma \\
\hline $\begin{array}{l}\text { Monolitik zirkonya } \\
\text { seramik } \\
\text { Zirconia Multi- } \\
\text { Layered Disc, } \\
\text { CAD-CAM Katana } \\
\text { (KAT) }\end{array}$ & $\begin{array}{l}\mathrm{ZrO}_{2} \text {, zirkonyum dioksit } \mathrm{Al}_{2} \mathrm{O}_{3} \\
\text { oranı } \% 0,1-0,05 \text { partikül } \\
\text { büyüklüğü 40nm } \\
\text { Tabakalı geçiş sistemi } \\
\% 35 \text { mine, } \% 15 \text { ilk geçiş } \\
\text { tabaka, } \% 15 \text { ikinci geçiş } \\
\text { tabaka, } \% 35 \text { dentin }\end{array}$ & $\begin{array}{l}\text { Noritake Dental } \\
\text { Supply Co., Ltd., } \\
\text { Miyoshi, Japan }\end{array}$ \\
\hline $\begin{array}{l}\text { Titanyum implant } \\
\text { (Nobel Replace } \\
\text { Implant System) }\end{array}$ & Titanyum alaşımı & $\begin{array}{l}\text { Nobel Biocare AB } \\
\text { Vastra Hamngatan } \\
\text { Göteborg, Sweden }\end{array}$ \\
\hline $\begin{array}{l}\text { Titanyum } \\
\text { abutment (Nobel } \\
\text { Replace abutment } \\
\text { System) }\end{array}$ & Titanyum alaşımı...................... & $\begin{array}{l}\text { Nobel Biocare AB } \\
\text { Vastra Hamngatan } \\
\text { Göteborg, Sweden }\end{array}$ \\
\hline
\end{tabular}

$\mathrm{ZrO}_{2}$, zirkonyum dioksit; $\mathrm{Al}_{2} \mathrm{O}_{3}$, alüminyum dioksit
Tablo 2. Materyallerin elastik modülüs değerleri ve poisson oranları

\begin{tabular}{|c|c|c|c|}
\hline Materyallerl & \begin{tabular}{|c|} 
Elastik \\
modülüs \\
(GPa)
\end{tabular} & $\begin{array}{c}\text { Poison } \\
\text { oranı }\end{array}$ & Referans ve yıl \\
\hline Katana & 210 & 0,35 & $\begin{array}{c}\text { Ha 2015; } \\
\text { de Kok ve ark.,2015 }\end{array}$ \\
\hline Nobel implant & 110 & 0,3 & $\begin{array}{l}\text { Chang ve ark., } 2010 \\
\text { Pessoa ve ark., } 2010\end{array}$ \\
\hline Nobel abutment & 110 & 0,3 & $\begin{array}{l}\text { Chang ve ark., } 2010 \\
\text { Pessoa ve ark.,2010 }\end{array}$ \\
\hline Kortikal kemik D2 & 13,7 & 0,3 & Baggi ve ark. 2008 \\
\hline Trabeküler kemik & 1,37 & 0,3 & Baggi ve ark. 2008 \\
\hline
\end{tabular}

Kemik modellenmesi için, erişkin bir hastanın mandibula çene kemiği, Konik Hüzme Işınlı Tomografide (ILUMA, Orthocad, CBCT, 3M Imtec, Oklahoma, USA) tarandı (Şekil 1). Taramada $120 \mathrm{kvp}, 3.8 \mathrm{~mA}$ 'de 40 saniyelik tarama ile 601 kesit elde edilmiştir. Daha sonra hacimsel veri $0.2 \mathrm{~mm}$ kesit kalınlığı ile rekonstrükte edilmiştir. DICOM 3.0 formatında export edilmiştir. Export edilen kesitler 3D- Doctor yazılımına alınmıştır (Şekil 1). 3 boyutlu model 3D-Doctor yazılımından .stl formatında export edilmiştir.

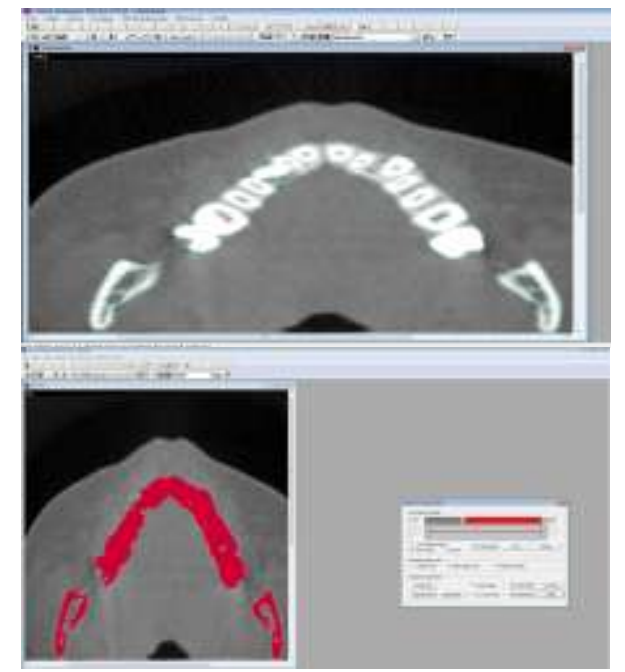

Şekil 1. Mandibular tam dişsiz kemik dokularının konik Hüzme Işınlı Tomografide modellenmesi

Mandibulada kortikal kemik, trabeküler kemik, implantlar, arayüzler (blood interface), abutmentlar, vidalar ve protez modele taşınmıştır. Rhinoceros yazılımında modeller 3 boyutlu uzayda doğru koordinatlarda modelleme tamamlanmıştır. 3 boyutlu koordinatlar korunarak Fempro yazilımına aktarılmıştır. Modellerde Bricks ve Tetrahedra elemanlar şeklinde katı modele çevrilmiştir. Bricks ve Tetrahedra katı modelleme sisteminde, Fempro modelde oluşturabildiği kadar 8 nodlu elemanlar kullanır. 8 nodlu elemanların gerekli detaya ulaşamadığı durumlarda 7 nodlu, 6 nodlu, 5 nodlu ve 4 
nodlu elemanlar kullanılır. 10'luk quadratic tetrahedral kullanılmıştır. Düğüm ve nod sayıları Tablo 3'de verilmektedir.

Tablo 3. Mandibular tam ark model tipleri, element ve nod sayıları

\begin{tabular}{|c|c|c|}
\hline $\begin{array}{l}\text { Mo } \\
\text { del }\end{array}$ & Model dizaynı & $\begin{array}{l}\text { Element } \\
\text { Ve nod } \\
\text { sayıları }\end{array}$ \\
\hline $\begin{array}{l}\text { Z- } \\
15, \\
5\end{array}$ & $\begin{array}{l}\text { Anterior iki implant lateral kesici dişler bölgesine, çift } \\
\text { taraflı ve dik olarak Posterior implantların apikal } \\
\text { kısımları } 2 \text {. premolar dişler hizasında servikal bölgesi } \\
\text { 1. Molar dişin mezial yarısı hizasında ve oklüzyon } \\
\text { düzlemine göre } 15^{\circ} \text { distale açılı } \\
\text { Kantilever uzunluğu } 5 \mathrm{~mm} \text {. } \\
\text { Tam ark sabit protez KAT seramik tek parça }\end{array}$ & $\begin{array}{l}882106 \\
181020\end{array}$ \\
\hline $\begin{array}{l}Z- \\
15, \\
9\end{array}$ & $\begin{array}{l}\text { Anterior iki implant lateral kesici dişler bölgesine, çift } \\
\text { taraflı ve dik } \\
\text { Posterior implantların apikal kısımları 2. premolar } \\
\text { dişler hizasında servikal bölgesi } 1 \text {. Molar dişin mezial } \\
\text { yarısı hizasında ve oklüzyon düzlemine göre } 15^{\circ} \\
\text { distale açılı } \\
\text { Kantilever uzunluğu } 9 \mathrm{~mm} \text {. } \\
\text { Tam ark sabit protez KAT seramik tek parça }\end{array}$ & $\begin{array}{l}874172 \\
181528\end{array}$ \\
\hline $\begin{array}{l}Z- \\
30, \\
5\end{array}$ & $\begin{array}{l}\text { Anterior iki implant lateral kesici dişler bölgesine, çift } \\
\text { taraflı ve dik } \\
\text { Posterior implantların apikal kısımları 2. premolar } \\
\text { dişler hizasında servikal bölgesi } 1 \text {. Molar dişin mezial } \\
\text { yarısı hizasında ve oklüzyon düzlemine göre } 30^{\circ} \\
\text { distale açlı } \\
\text { Kantilever uzunluğu } 5 \mathrm{~mm} \text {. } \\
\text { Tam ark sabit protez KAT seramik tek parça }\end{array}$ & $\begin{array}{l}884556 \\
181299\end{array}$ \\
\hline $\begin{array}{l}Z- \\
30, \\
9\end{array}$ & $\begin{array}{l}\text { Anterior iki implant lateral kesici dişler bölgesine, çift } \\
\text { taraflı ve dik } \\
\text { Posterior implantların apikal kısımları } 2 \text {. premolar } \\
\text { dişler hizasında servikal bölgesi } 1 \text {. Molar dişin mezial } \\
\text { yarısı hizasında ve oklüzyon düzlemine göre } 15^{\circ} \\
\text { distale açılı } \\
\text { Kantilever uzunluğu } 9 \mathrm{~mm} \text {. } \\
\text { Tam ark sabit protez KAT zirkonya seramik tek parça }\end{array}$ & $\begin{array}{l}875430 \\
182008\end{array}$ \\
\hline
\end{tabular}

Çalışmada 4 adet mandibular matematiksel model planlanmıştır. $2 \mathrm{~mm}$ eksternal kortikal kemik kalınlığı ve $1 \mathrm{~mm}$ trabeküler kemik kalınlığı ve rezidüel alveoler kret yüksekliği total olarak $22 \mathrm{~mm}$ olarak parametreler düzenlenmiştir (Şekil 2).

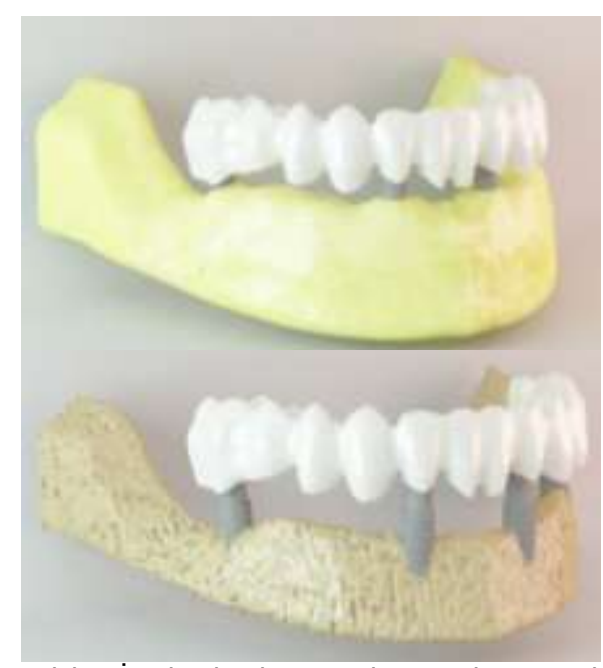

Şekil 2. İmplantlar ile seramik protetik üst yapıların kortikal ve trabeküler kemik bilgisayar destekli dizayn sonlu elemanlar model görüntüleri

\section{İmplantların ve protetik üstyapıların modellenmesi}

Bu çalışmada all on four implant tarasımlarında Nobel Biocare implant ve abutmentları kullanılmıştır (Nobel Replace Implant System-Nobel Biocare). Bütün implantların çapları $4 \mathrm{~mm}$ ve anterior implant uzunlukları $11,5 \mathrm{~mm}$, posterior implant uzunlukları 13 $\mathrm{mm}$ olarak seçilmiştir. Abutmentlar $4 \mathrm{~mm}$ çapında ve 8 mm uzunluğunda tespit edilmiştir.

3 boyutlu ağ yapısının düzenlenmesi ve daha homojen hale getirilmesi, 3 boyutlu katı modelin oluşturulması ve sonlu elemanlar stres analizi işlemi için Intel Xeon \& R CPU 3,30 GHz işlemci, $500 \mathrm{gb}$ Hard disk, 14 GB RAM donanımlı ve Windows 7 Ultimate Version Service Pack 1 işletim sistemi olan bilgisayardan, Activity 880 (smart optics Sensortechnik GmbH, Sinterstrasse 8, D-44795 Bochum, Almanya) optik tarayıcısı ile 3 boyutlu tarama cihazından, Rhinoceros 4.0 (3670 Woodland Park Ave N ,Seattle, WA 98103 USA) 3 boyutlu modelleme yazılımından, VRMesh Studio (VirtualGrid Inc, Bellevue City, WA, USA) ve Algor Fempro (ALGOR, Inc. 150 Beta Drive Pittsburgh, PA 15238-2932 USA) analiz programından yararlanılmıştır.

$\mathrm{Bu}$ verilere göre modeller oluşturuldu. CADCAM köprü protezi için dişlerin boyutları ve morfolojileri Wheeler's study (Nelson 2009) referans alınarak tespit edilmiştir. Kron kalınlığı 5-6 mm yüksekliği $10 \mathrm{~mm}$ olarak ayarlanmıştır ve gövdeler ridge-lap oluşturulmuştur (Şekil 3). 3D-FEA modelller Marc 2013 (MSC Software Corporation, Santa Ana, CA, USA) bilgisayar yazılımı (computer software) kullanılarak ve bilgisayarlı tomografi (CT) kesitlerine göre oluşturulmuştur. Bütün modellerde molarlar arası ark mesafesi $78 \mathrm{~mm}$ olarak tespit edilip $5 \mathrm{~mm}$ ve $9 \mathrm{~mm}$ uzunluklarda iki farklı kantilever uzunluğu seçilmiştir.

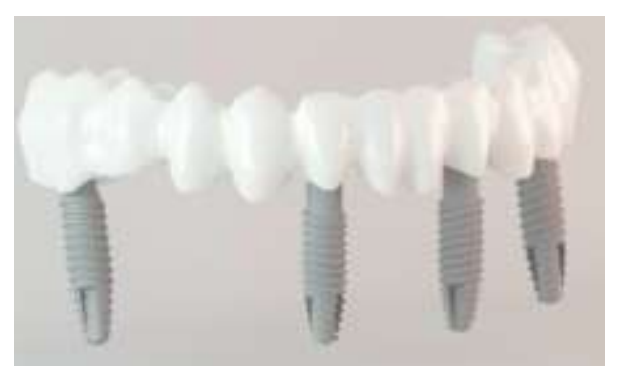

Şekil 3. Monolitik zirkonya sabit protez modeli

Abutmentın merkezinden yapılan ölçümlerde, 5 mm kantilever kullanılan modeller için; L1 (anterior implantlar arası mesafe) $=13,6 \mathrm{~mm}$ ve $\mathrm{L} 2$ (posterior 
implantlar arası mesafe) $=46 \mathrm{~mm}$ olarak tespit edilmiştiir. $9 \mathrm{~mm}$ kantilever kullanılan modeller için L1 (anterior implantlar arası mesafe) $=13,6 \mathrm{~mm}$ ve $\mathrm{L} 2$ $=40,6 \mathrm{~mm}$ olarak tespit edilmiştir. Mezial ve distal implanlar arası mesafe (ark şeklinde ölçüldü); $9 \mathrm{~mm}$ kantilever model $=19,6 \mathrm{~mm}$, ve $5 \mathrm{~mm}$ kantilever model $=24,5 \mathrm{~mm}$ olarak ölçülmüştür.

\section{Sistemin birleştirilmesi}

İmplantlar, abutmentler, CAD-CAM protez materyalleri ve çevre dokular arasındaki bağlantılar, yükü devamlı transfer etmek için tasarlanmıştır. Kemik ve implantlar arasında \%100 osseointegrasyon varsayıldı ve benzer çalışmalarla uyumlu olarak implantların vestibüler ve palatal yüzeyleri için $1 \mathrm{~mm}$ kemik kalınlığı belirlendi. Bu çalışmada D2 tipi kemik modeli kullanılmıştır. ${ }^{23,24}$

\section{Materyal Özellikleri}

Tüm modeller lineer, homojen ve izotropik materyaller olarak kabul edilmiştir. Homojen olması, mekanik özelliklerinin yapısal olarak her elemanda benzer olduğunu gösterir. İzotropik, yapısal elemanın her yönde materyal özelliklerinin aynı olduğu durumu tanımlamaktadır. Linear elastisite; deformasyon veya gerilim (strain) uygulanan kuvvetler altında oransal olarak değişkenlik göstermesidir. Mandibula modelinin dış sınırları boyunca arka ve alt yüzeylerinden serbestlik derecesi (degree of freedom-DOF) sıfır hareket olacak şekilde tespit edilmiştir (Şekil 4B).

\section{Yükleme Koşulları}

Uygulanan kuvvetler, Ferrario ve ark. tarafından yapılan çalışmada kadın ve erkek hastaların dişlerine gelen kuvvetlerin ortalamaları alınarak seçilmiştir. $^{25}$ 1. Molar oblik yükleme, sağ kantilever uzantısı olan 1. Molar dişin lingual tüberkülünün bukkal eğimine ve oklüzyon düzlemine göre $30^{\circ}$ açılı $150 \mathrm{~N}$ oblik kuvvet uygulanmıştır (Şekil 4A).

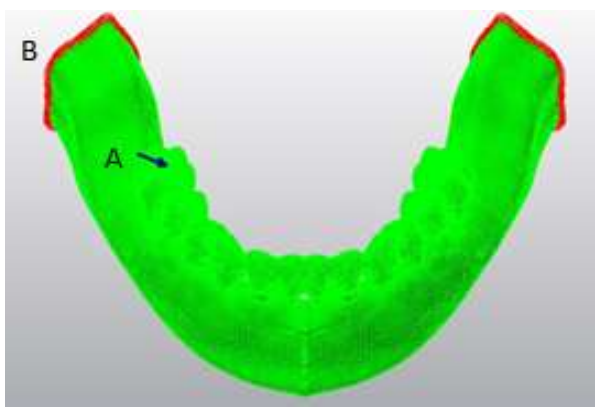

Şekil 4. A; Yükleme koşulu, sağ kantilever $30^{\circ}$ oblik yükleme $150 \mathrm{~N}$.

B; mandibular modelin dış sınırları kırmızı çizgiler ile gösterilmekte

\section{D-FEA sonuçlarının değerlendirilmesi}

Streslerin yoğun olarak gözlendiği alanlarda noktasal değerler belirlendi. Stres dağılımlarını gösteren görüntülerin sol üst tarafındaki skaladan renklere göre stres değerlerinin dağılımı görülebilmektedir. Şekillerde her renk bir stres aralığını (MPa) temsil etmektedir. Resimlerde sol üst köşelerde yer alan skaladaki renklere göre, von Mises stres değerleri ve çekme stresleri maviden kırmızıya doğru artmaktadır. Basma stresleri ise negatif değerler ile gösterilmekte ve mutlak değeri kullanılmaktadır. Basma stresleri için mavi değerler daha yüksek stresleri belirtmektedir.

3D-FEA ile elde edilen veriler bilgisayar sisteminde varyansı olmayan matematiksel hesaplamalar oldukları için istatistiksel değerlendirmeler yapılamamaktadır. Kesit görünümleri, düğümlerdeki stres miktarları ve stres dağılımları değerlendirilmekte ve yorumlanmaktadır.

\section{BULGULAR}

Çalışmada 4 farklı modelde, 1.molar oblik yüklemede; kortikal kemikte, trabeküler kemikte, implantlarda, abutmentlarda ve protetik yapılarda oluşan çekme, basma ve Von Mises stresleri değerlendirildi.

\section{değerleri}

Kortikal kemikte oluşan çekme stres Z-15,5: 12,46 MPa; Z-15,9: 14,11 MPa; Z30,5: 14,78 MPa; Z-30,9: 16,6 MPa elde edilmiştir (Tablo 4). Buna göre en yüksek stres değerleri Z-30,9 modelde en düşük değerler Z-15,5 modelde gözlendi (Şekil 5).

Tablo 4. Kortikal kemikte çekme stres değerleri (Pmax-MPa)

\begin{tabular}{|c|c|c|c|c|c|c|c|}
\hline \multicolumn{4}{|c|}{ 1.model $(Z-15,5)$} & \multicolumn{4}{|c|}{ 3.model $(Z-30,5)$} \\
\hline Distal & Mezial & Mezial & Distal & Distal & Mezial & Mezial & Distal \\
\hline 12,46 & 3,29 & 1,72 & 1,83 & 14,78 & 5,02 & 1,53 & 1,54 \\
\hline \multicolumn{4}{|c|}{ 2.model $(Z-15,9)$} & \multicolumn{4}{|c|}{ 4.model $(Z-30,9)$} \\
\hline Distal & Mezial & Mezial & Distal & Distal & Mezial & Mezial & Distal \\
\hline 14,11 & 4,67 & 1,13 & 1,46 & 16,6 & 3,54 & 1,53 & 1,38 \\
\hline
\end{tabular}

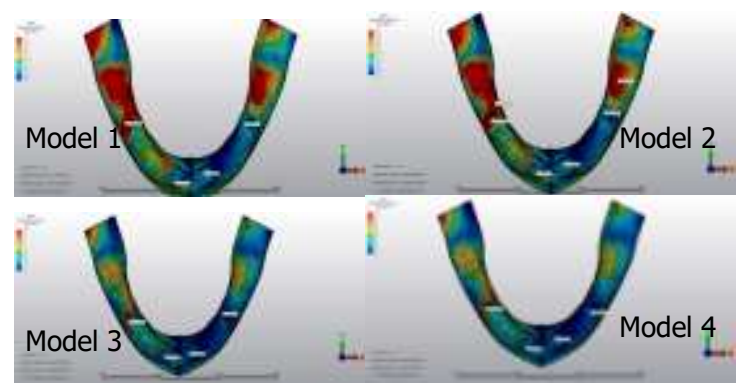

Şekil 5. 1-4. modellerde kortikal kemik çekme stres değerleri (Pmax-MPa) 
Trabeküler kemikte oluşan çekme stres değerleri

Z-15,5: 2,15 MPa; Z-15,9: 2,22 MPa; Z-30,5: 2,31 MPa; Z-30,9: 3,19 MPa elde edilmiştir (Tablo 5). Buna göre en yüksek stres değerleri Z-30,9 modelde en düşük değerler Z-15,5 gözlendi (Şekil 6).

Tablo 5. Trabeküler kemikte çekme stres değerleri (Pmin$\mathrm{MPa})$

\begin{tabular}{|c|c|c|l|l|l|l|l|}
\hline \multicolumn{2}{|c|}{ 1.model (Z-15,5) } & \multicolumn{4}{|c|}{ 3.model (Z-30,5) } \\
\hline Distal & Mezial & Mezial & Distal & Distal & Mezial & Mezial & Distal \\
\hline 2,15 & 0,39 & 0,17 & 0,45 & 2,31 & 0,41 & 0,17 & 0,73 \\
\hline 2.model (Z-15,9) & & $\mathbf{4 . m o d e l} \mathbf{( Z - 3 0 , 9 )}$ & \\
\hline Distal & Mezial & Mezial & Distal & Distal & Mezial & Mezial & Distal \\
\hline 2,22 & 0,49 & 0,13 & 0,48 & 3,19 & 0,51 & 0,19 & 0,39 \\
\hline
\end{tabular}

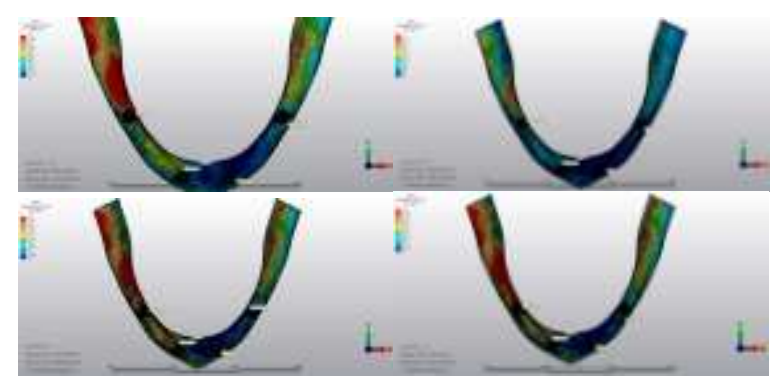

Şekil 6. 1-4. modellerde trabeküler kemik çekme stres değerleri (Pmax-MPa)

\section{değerleri}

Kortikal kemikte oluşan basma stres

Z-15,5: -18,56 MPa; Z-15,9: -19,15 MPa; Z30,5: -19,81 MPa; Z-30,9: -20,55 MPa elde edilmiştir (Tablo 6). Buna göre en yüksek stres değerleri Z-30,9 modelde en düşük değerler Z-15,5 modelde gözlendi (Şekil 7).

Tablo 6. Kortikal kemikte basma stres değerleri (Pmin-MPa)

\begin{tabular}{|c|c|c|c|c|c|c|c|}
\hline \multicolumn{4}{|c|}{ 1.model $(\mathrm{Z}-15,5)$} & \multicolumn{4}{|c|}{ 3.model $(Z-30,5)$} \\
\hline Distal & Mezial & Mezial & Distal & Distal & Mezial & Mezial & Distal \\
\hline$-18,56$ & $-2,43$ & $-3,07$ & $-3,69$ & $-19,81$ & $-2,16$ & $-3,14$ & $-3,27$ \\
\hline \multicolumn{4}{|c|}{ 2.model $(Z-15,9)$} & \multicolumn{4}{|c|}{ 4.model $(Z-30,9)$} \\
\hline Distal & Mezial & Mezial & Distal & Distal & Mezial & Mezial & Distal \\
\hline$-19,15$ & $-2,15$ & $-2,49$ & $-4,96$ & $-20,55$ & $-2,17$ & $-2,8$ & $-4,89$ \\
\hline
\end{tabular}

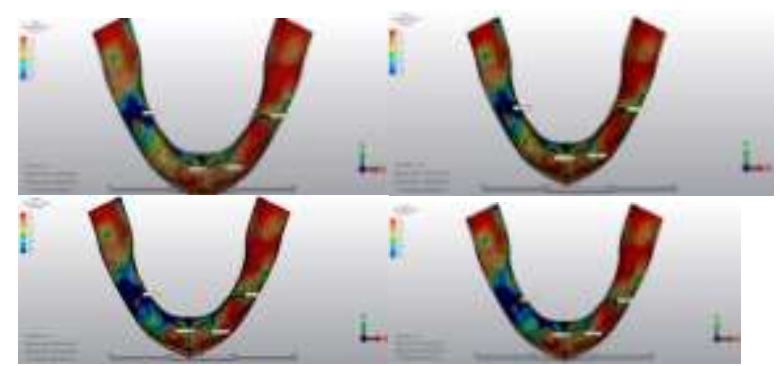

Şekil 7. 1-4. modellerde kortikal kemik basma stres değerleri (Pmin-MPa)

\section{Trabeküler kemikte oluşan basma stres} değerleri

Z-15,5: -1,04 MPa; Z-15,9: -1,25 MPa; Z-30,5: -1,23 MPa; Z-30,9: -1,94 MPa elde edilmiştir (Tablo 7). Buna göre en yüksek stres değerleri Z-30,9 modelde en düşük değerler Z-15,5 modelde gözlendi. (Şekil 8).

Tablo 7. Trabeküler kemikte basma stres değerleri (Pmin$\mathrm{MPa})$

\begin{tabular}{|l|l|l|l|l|l|l|l|}
\hline \multicolumn{1}{|l|}{ 1.model (Z-15,5) } & \multicolumn{4}{|c|}{ 3.model (Z-30,5) } \\
\hline Distal & Mezial & Mezial & Distal & Distal & Mezial & Mezial & Distal \\
\hline$-1,04$ & $-0,91$ & $-0,42$ & $-0,44$ & $-1,23$ & $-0,9$ & $-0,44$ & $-0,85$ \\
\hline 2.model (Z-15,9) & & \multicolumn{4}{|c|}{ 4.model (Z-30,9) } \\
\hline Distal & Mezial & Mezial & Distal & Distal & Mezial & Mezial & Distal \\
\hline$-1,25$ & $-0,92$ & $-0,51$ & $-0,75$ & $-1,94$ & $-0,92$ & $-0,5$ & $-0,95$ \\
\hline
\end{tabular}

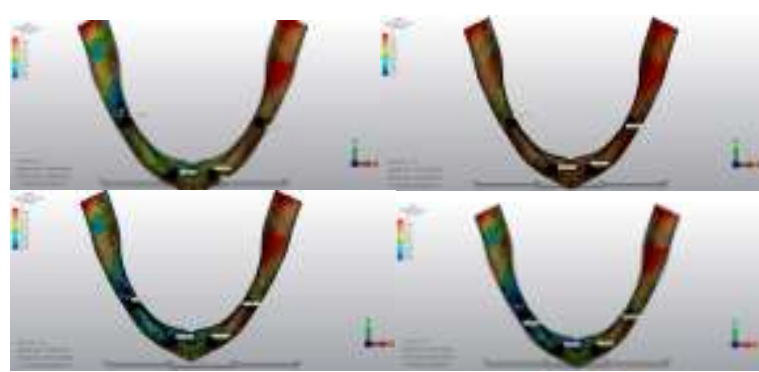

Şekil 8. 1-4. modellerde trabeküler kemik basma stres değerleri (Pmin-MPa)

\section{Anterior ve Posterior implantlarda von Mises stres değerleri}

Anterior implantlarda von Mises stres değerleri: İmplant von Mises stresleri; mezial implantlar anterior implantları belirtmektedir. Z-15,5: 15,93 MPa; Z-15,9: 20 MPa; Z-30,5: 17,86 MPa; Z-30,9: 22,72 MPa elde edildi (Tablo 8). Buna göre en yüksek stres değerleri Z-30,9 modelde, en düşük ise Z-15,5 modelde gözlendi (Şekil 9).

Posterior implantlarda von Mises stres değerleri: İmplant von Mises stresleri; distal implantlar posterior implantları belirtmektedir. Z-15,5: 47,28 MPa; Z-15,9: 55,17 MPa; Z-30,5: 25,05 MPa; Z-30,9: 30,54 MPa elde edilmiştir (Tablo 8). En yüksek stres değeri Z-15,9 modelde en düşük ise Z-30,5 modelde izlendi (Şekil 9).

Tablo 8. Anterior ve posterior implantlarda elde edilen von Mises stres değerleri (MPa)

\begin{tabular}{|l|l|l|l|l|l|l|l|}
\hline \multicolumn{3}{|l|}{ 1.model (Z-15,5) } & \multicolumn{4}{|c|}{ 3.model (Z-30,5) } \\
\hline Distal & Mezial & Mezial & Distal & Distal & Mezial & Mezial & Distal \\
\hline 47,28 & 15,93 & 0,19 & 0,2 & 25,05 & 17,86 & 9,53 & 3,06 \\
\hline 2.model (Z-15,9) & \multicolumn{2}{|l|}{ 4.model (Z-30,9) } \\
\hline Distal & Mezial & Mezial & Distal & Distal & Mezial & Mezial & Distal \\
\hline 55,17 & 20 & 9,56 & 3,96 & 30,54 & 22,72 & 9,65 & 2,59 \\
\hline
\end{tabular}




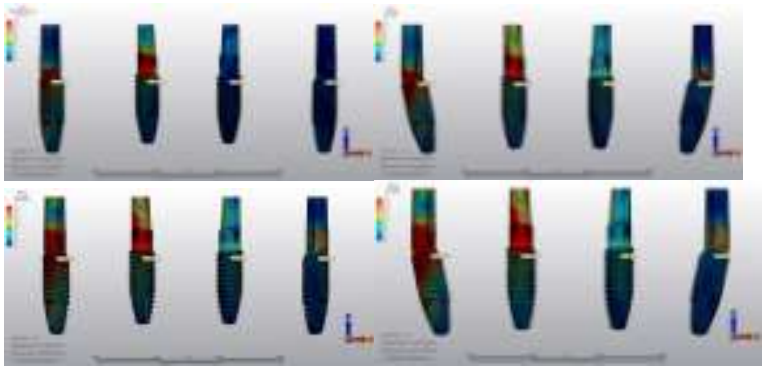

Şekil 9. 1-4. modellerde implantlarda maksimum von Mises stres değerleri (MPa)

Üst yapılara ait von Mises stres değerleri Z-15,5: 1592,3 MPa; Z-15,9: 919,8 MPa; Z30,5: 1592,3 MPa; Z-30,9: 919,8 MPa elde edilmiştir. Buna göre en yüksek stres değerleri $Z-15,5$ ve $Z-30,5$ modellerde, en düşük değerler $Z-15,9$ ve $Z-30,9$ modelde gözlendi. En yüksek stres değerleri 1. moların okluzal yüzünde yoğunlaştı (Şekil 10).

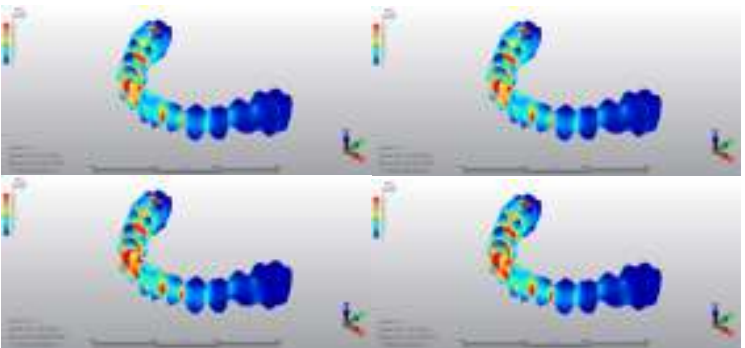

Şekil 10. 1-4. modellerde üstyapılarda maksimum von Mises stres değerleri (MPa)

\section{TARTIŞMA}

2 hipotez şunlardır: iki farklı kantilever uzunluğunun restorasyon, kemik veya implantlarda oluşturdukları stres değerleri benzerdir; ve farklı posterior implant açılanmaları biyomekanik olarak stres iletiminde önemli farklılık oluşturmamaktadır. Bulgular ışığındaa ilk hipotez reddedilirken ikinci hipotez kısmen reddedilmiştir.

Literatür incelemesinde genellikle $2 \mathrm{~mm}$ kalınlıkta ve tip 2 kortikal kemik üzerinde implant çalışmaları olduğu gözlenmiştir. ${ }^{21,26}$ Yapılan bir çalışmada kortikal kemik kalınlığı arttıkça stres değerlerinde azalma olduğu, kalınlık azaldıkça stres değerlerinin arttığı bulgulanmıştır. ${ }^{26}$ Çalışmamızın benzer çalışmalar ile karşılaştırıması bakımından bu değerler tercih edilmiştir.

Kantilever okluzyon modelinde, vertikal yüklemede kuvvetlerin tolere edilebildiği ancak oblik yüklemelerde yıkıcı gerilimler oluştuğu belirtildiği için sadece oblik yükleme kullanılmıştır. ${ }^{27}$ Bukkolingual yönde tek taraflı olarak $30^{\circ}$ açı ile 1. molar dişin santral fossasından $150 \mathrm{~N}$ kuvvet uygulanmıştır.

In vivo ve in vitro çalışmalarda all-on-four implant konseptinde implant çapları anterior implantlar için genellikle 3,75-4 $\mathrm{mm}$ posterior implantlar için 44,3 mm olarak kullanılmaktadır. ${ }^{28-31}$ Diğer çalışmalar ile karşılaştırılması ve yaygın kullanımı nedeni ile çalışmamızda $4 \mathrm{~mm}$ çapında anterior ve posterior implantlar tercih edilmiştir.

Peri-implant gerilim ve gerilmelerde kantilever uzunluğunun etkili olduğu belirtilmektedir. Bellini ve arkadaşlarının $100 \mathrm{~N}$ kantilever yüklemesi yapılan allon-four modellerde açılı implantlarda kemikteki stresi analiz ettikleri çalışmalarında minimum asal gerilme stresleri $15 \mathrm{~mm}$ kantilever uzantısında -24 MPa tespit etmiş̧lerdir. Kantilever uzunluğu 5 mm'den 15 mm'ye çıkarıldığı zaman minimum asal gerilme stresleri \% 33 oranında artmıştır. ${ }^{32}$ Başka bir çalışmada, kortikal kemikteki maksimum stresler kantilever olan modellerde 1,5 kat daha yüksek bulunmuştur. ${ }^{33} \mathrm{Bu}$ çalışmada da tüm yükleme koşullarında kantilever uzunluğu 5 mm'den 9 mm'ye çıkarıldığı zaman maksimum ve minimum asal gerilme değerleri artmıştır.

Monolitik zirkonya seramikler günümüzde implant üstü sabit protetik restorasyonlarda kullanım alanları bulmaktadır. ${ }^{34}$ Monolitik zirkonya seramik sistemleri tam ark sabit restorasyonlarda kullanılmasına rağmen literatür incelemesinde all-on-four implant destekli restorasyonlarda kullanımına rastlanmamıştır.

All-on-four implant destekli sabit protetik restorasyonlarda tam ark tek parça monolitik zirkonya seramik kullanılarak yapılan bir analiz çalışması bulunmadığı için karşılaştırmalar kendi çalışma planlamalarımız ile yapılmaktadır. Monolitik zirkonya seramiklerde yoğun stresler oluşurken implant ve kemiğe iletilen streslerde azalma görülmüştür. Çalışmamızda kortikal ve trabeküler kemikteki maksimum ve minimum asal gerilme değerleri monolitik zirkonya seramik köprü protezlerinden daha yüksek bulunmuştur.

Daha sert ve dayanıklı materyallerin biyomekanik olarak daha tercih edilebilir olduğu belirtilmiştir. Ayrıca all-on-four tedavi planlamasında stres değerlerinin kemik direnci sınırlarını aşmadığı bulunmuştur. ${ }^{35}$

All-on-four mandibular implant tasarımında implantların yerleştirilmesini takiben bir hafta içinde akrilik rezin kaide ve dişlerden oluşan geçici protez yapılarak implantlar hemen yüklenmekte ve fonksiyona girmektedir. Daimi protez ise yaklaşık üç ay sonra metal-seramik tam ark sabit protez şeklinde yapılmak- 
tadır. ${ }^{36,37}$ Araştırmamızda tam ark seramik protez yapılması planlandığı için erken yükleme değil implantların etrafında matür kemiğin olduğu tam iyileşme dönemi simüle edilmiştir. Bu nedenle immatür kemik değerleri kullanılmamıştır.

Kortikal kemikte meydana gelen maksimum asal gerilme değerleri 100-130 MPa minimum asal gerime değerleri 170-190 MPa trabeküler kemikte meydana gelen maksimum ve minimum asal gerilme değerleri de $5 \mathrm{MPa}$ değerinden fazla olduğu zaman kemikte aşırı yükleme olduğu savunulmaktadır. ${ }^{21}$ Belirtilen yükleme koşulunda elde edilen stres değerleri sınırlar dahilinde kalmıştır.

Sonlu elamanlar stres analizlerinde elastik modülüsü yüksek olan kortikal kemik streslerin büyük miktarını karşılaması nedeniyle trabeküler kemikte daha düşük stres değerlerinin tespit edilmesine neden olmaktadır. ${ }^{21,38}$ Çalışmamızdaki tüm modellerde, her yükleme koşulunda, en yüksek stres değerleri implantlarda ve kortikal kemikte, en düşük stres değerleri ise trabeküler kemikte elde edilmiştir.

Çalışmamızın limitasyonlarında sonlu elemanlar stres analizine yönelik belirttiğimiz sınırlamaları sayılabilir. Mandibular modeller lineer, elastik ve homojen olarak kabul edilmektedir. Ancak mandibula kemik yapısı homojen özellikte değil ve anizotropik yapısından dolayı farklı gerilim dağılımları olmaktadır. ${ }^{39}$ İmplant ve kemik arasında tam osseointegrasyon olduğu kabul edilmekte ancak klinik olarak implant ve kemik arasında parsiyel kontakt ve osseointegrasyon olduğu bildirilmektedir. ${ }^{28,40}$ İmplantlara immediat yükleme yapılmaması ve kas kuvvetlerinin etkisinin değerlendirilmeye alınmaması çalışmanın diğer bir limitasyonudur. Farklı yükleme koşullarında stres dağılım düzeylerinin tespiti yapılacak ikinci bölüm çalışma da planlanmaktadır. Bu çalışmaları takip eden daha ileri in vitro ve in vivo çalışmaların yapılması gerekmektedir. Özellikle protetik rehabilitasyon bakımından kullanılan son teknoloji seramik sistemlerinin kullanıldığı çalışmalara ihtiyaç bulunmaktadır.

\section{SONUÇLAR}

$\mathrm{Bu}$ in vitro çalışmanın bulgularına dayanarak, aşağıdaki sonuçlar çıkarılmıştır:

1. Posterior implant çevresi kortikal kemikte; monolitik zirkonya seramik köprü protezlerinde yüksek çekme ve basma stresleri meydana gelmiştir.

2. Posterior implant açılanması $15^{\circ}$ ve $30^{\circ}$ olan tüm modeller arasında kortikal kemikte çekme ve basma stres değerleri bakımından anlamlı fark bulunamamıştır.

3. Posterior implant açılanması $15^{\circ}$ den $30^{\circ}$ düzeyine çıktığında implantlardaki von Mises gerilme değerlerinde azalma görülmüştür. İmplantlarda von Mises gerilmeleri tüm yüklemelerde posterior implantların boyun bölgesinde 2 . ve 3 . yivinde yoğunlaşmıştır.

NOT: Maddi destek ve çıkar ilişkisi

Bu çalışma Afyon Kocatepe Üniversitesi Bilimsel

Araştırma Projeleri Komisyonu tarafindan 17.DUS.01

proje numarası ile desteklenmiştir. Yazarların çıkara

dayalı bir ilişkisi yoktur.

\section{KAYNAKLAR}

1. Durkan R, Deste G, Şimşek H. Monolitik zirkonya seramik sistemlerinin üretim tipleri ile aşınma, optik ve estetik özellikleri. Atatürk Üniv Diş Hek Fak Derg 2018; 28:263-70.

2. Sulaiman TA, Abdulmajeed AA, Donovan TE, Ritter AV, Lassila LV, Vallittu PK, Närhi TO. Degree of conversion of dual-polymerizing cements light polymerized through monolithic zirconia of different thicknesses and types. J Prosthet Dent 2015; 114:103-8.

3. Zhang F, Vanmeensel K, Batuk M, Hadermann J, Inokoshı M, Van Meerbeek B, Naert I, Vleugels J. Highly-translucent, strong and aging-resistant 3YTZP ceramics for dental restoration by grain boundary segregation. Acta Biomater 2015; 16:215-22.

4. Ilie N, Stawarczyk B. Quantification of the amount of blue light passing through monolithic zirconia with respect to thickness and polymerization conditions. J Prosthet Dent 2015; 113:114-21.

5. Ueda K, Guth JF, Erdelt K, Stimmelmayr M, Kappert $H$, Beuer F. Light transmittance by a multicoloured zirconia material. Dent Mater J 2015; 34:310-4.

6. Katana. Multilayered zirconia now available in new shades. www.dentalproductsreport.com. (2015). Erişim tarihi:4.08.2016.

7. Misch, C.E. Dental İmplant Protezler. (Çev. Kutay Ö.). 1. Basım, İstanbul, Nobel Matbaacilık, 2009; 32:253-4.

8. Van Lierde KM, Browaeys $H$, Corthals $P$, Matthys $C$, Mussche $P$, Van Kerckhove E, De Bruyn H. Impact of fixed implant prosthetics using the 'all-onfour' treatment concept on speech intelligibility, 
articulation and oromyofunctional behaviour. Int J Oral Maxillofac Surg 2012; 41:1550-7.

9. Spinelli D, Ottria L, De Vico G, Bollero R, Barlattani A, Bollero $P$. Full rehabilitation with Nobel clinician $($ ) and procera implant bridge $\AA$ : Case report. Oral Implantol (Rome) 2013; 6:25-36.

10. Ehsani S, Siadat $H$, Alikhasi M. Comparative evaluation of impression accuracy of tilted and straight implants in All-on-Four technique. Implant Dent 2014; 23:225-30.

11. Dellavia C, Francetti L, Rosati R, Corbella S, Ferrario VF, Sforza C. Electromyographic assessment of jaw muscles in patients with All-onFour fixed implant-supported prostheses. J Oral Rehabil 2012; 39:896-904.

12. Taruna M, Chittaranjan B, Tella S, Abuusaad MD. Prosthodontic perspective to all-on-4 concept for dental implants. J Clin Diagn Res 2014; 8:16-9.

13. Jensen OT, Adams MW, Cottam JR, Parel SM, Phillips WR. The all on 4 shelf: Mandible. Journal of Oral and Maxillofacial Surgery, 2011; 69:175-81.

14. Kurşunoğlu S. İmplant boyu ve çapının yükleme sonrası oluşan rezorpsiyona etkisinin 3 boyutlu sonlu eleman stres analiz yöntemi ile incelenmesi. Doktora tezi, Ankara Üniversitesi Sağlık Bilimleri Enstitüsü, 2011

15. Adıgüzel Ö. Sonlu elemanlar analizi: Derleme. Dicle Diş hek Derg 2010; 11:18-23.

16. Öztürk B. Farklı implant-abutment bağlantı tasarımlarında oluşan streslerin ve hareket serbestliğinin; 3 boyutlu sonlu elemanlar stres analiz yöntemi ile incelenmesi. Doktora Tezi, Ankara Üniversitesi Sağlık Bilimleri Enstitüsü, 2015.

17. Ha SR. Biomechanical three-dimensional finite element analysis of monolithic zirconia crown with different cement type. J Adv Prosthodont 2015; 7:475-83.

18. de Kok P, Kleverlaan $C$, de Jager N, Kuijs $R$, Feilzer AJ. Mechanical performance of implantsupported posterior crowns. J Prosthet Dent 2015; 114:59-66.

19. Chang $\mathrm{CH}$, Chen $\mathrm{CS}$, Hsu $\mathrm{ML}$, Dent $\mathrm{M}$. Biomechanical effect of platform switching in implant dentistry: A three-dimensional finite element analysis. Int J Oral Maxilofac Implants 2010; 25:295-304.

20. Pessoa RS, Muraru L, Júnior EM, Vaz LG, Sloten JV, Duyck J, Jaecques SV. Influence of implant connection type on the biomechanical environment of immediately placed implants - CT-based nonlinear, three-dimensional finite element analysis. Clin Implant Dent Relat Res 2010; 12: 219-34.

21. Baggi L, Cappelloni I, Girolama MD, Maceri F, Vairo $\mathrm{G}$. The influence of implant diameter and length on stress distribution of osseointegrated implants related to crestal bone geometry: A threedimensional finite element analysis. J Prosthet Dent 2008; 100:422-31.

22. Fanuscu MI, Iida K, Caputo AA, Nishimura RD. Load transfer by an implant in a sinus-grafted maxillary model. Int $\mathrm{J}$ Oral Maxillofac Implants 2003; 18:667-74.

23. Fanuscu MI, Vu HV, Poncelet B. Implant biomechanics in grafted sinus: a finite element analysis. J Oral Implantol 2004; 30:59-68.

24. Huang HL, Fuh LJ, Ko CC, Hsu JT, Chen CC. Biomechanical effects of a maxillary implant in the augmented sinus: a three-dimensional finite element analysis. Int J Oral Maxillofac Implants 2009; 24:455-62.

25. Ferrario VF, Sforza C, Serrao G, Dellavia C, Tartaglia GM. Single tooth bite forces in healthy young adults. J Oral Rehabil 2004; 31:18-22.

26. Medetalibeyoğlu F, Kaymaz I, Korkmaz IH, Dağsuyu IM, Akpınar N. Mini vida yerleştirilmiş mandibulada kortikal kemik kalınlığına bağlı olarak gerilmelerin incelenmesi. Sakarya Ü Fen Bilimleri Enst Derg 2012; 16:294-302.

27. Doğan DO, Polat NT, Polat S, Şeker E, Gül EB. Evaluation of all on four concept and alternative design with 3D finite element analysis method. Clinical Implant Dent And Related Res 2012; 16:501-10.

28. Takahashi T, Shimamura I, Sakurai K. Influence of number and inclination angle of implants on stress distribution in mandibular cortical bone with All-on4 Concept. J Prosthodont Res 2010; 54:179-84.

29. Maló P, De Araújo Nobre M, Lopes A, Moss SM, Molina GJ. A longitudinal study of the survival of All-on-four implants in the mandible with up to 10 years of follow-up. JADA 2011; 142:310-20.

30. Barão VA, Delben JA, Lima J, Cabral T, Assunção WG. Comparison of different designs of implantretained overdentures and fixed full-arch implantsupported prosthesis on stress distribution in edentulous mandible-a computed tomographybased three-dimensional finite element analysis. J Biomech 2013; 46:1312-20. 
31. Li X, Cao Z, Qıu X, Tang Z, Gong L, Wang D. Does matching relation exist between the length and the tilting angle of terminal implants in the all-on-four protocol? Stress distributions by 3D finite element analysis. J Adv Prosthodont 2015; 7:240-8.

32. Bellini CM, Romeo D, Galbusera F, Taschieri S, Raimondi MT, Zampelis A, Francetti L. Comparison of tilted versus nontilted implant-supported prosthetic designs for the restoration of the edentuous mandible: A biomechanical study. Int J Oral Maxillofac Implants 2009; 24:511-7.

33. Stegaroiu R, Khraisat A, Nomura S, Miyakawa O. Influence of superstructure materials on strain around an implant under 2 loading conditions: A technical investigation. Int J Oral Maxillofac Implants 2004; 19:735-42.

34. Zhao K, Pan Y, Guess PC, Zhang XP, Swain MV. Influence of veneer application on fracture behavior of lithium-disilicate-based ceramic crowns. Dental Materials, 2012; 28:653-60.

35. Bhering CL, Mesquita MF, Kemmoku DT, Noritomi PY, Consani RL, Barão VA. Comparison between all-on-four and all-on-six treatment concepts and framework material on stress distribution in atrophic maxilla: A prototyping guided 3D-FEA study. Mater Sci Eng C Mater Biol Appl 2016; 69:715-25.

36. Maló P, Rangert B, Nobre M. All-on-Four" immediate-function concept with Brånemark system implants for completely edentulous mandibles: a retrospective clinical study. Clin Implant Dent Relat Res 2003; 5:2-9.

37. Agliardi E, Panigatti S, Clericò M, Villa C, Malò P. Immediate rehabilitation of the edentulous jaws with full fixed prostheses supported by four implants: Interim results of a single cohort prospective study. Clin Oral Implants Res 2010; 21:459-65.

38. Meijer HJ, Kuiper JH, Starmans FJ, Bosman F. Stress distribution around dental implants: influence of superstructure, length of implants and height of mandible. J Prosthet Dent 1992; 68:96102.

39. Geng JP, Tan KB, Liu GR. Application of finite element analysis in implant dentistry: A review of the literature. J Prosthet Dent 2001; 85: 585-98.

40. Zampelis A, Rangert B, Heijl L. Tilting of splinted implants for improved prosthodontic support: A two-dimensional finite element analysis. J Prosthet Dent 2007; 97:35-43.

\section{Yazışma Adresi}

Doç. Dr. Rukiye DURKAN

Afyonkarahisar Sağlık Bilimleri Üniversitesi, Diş Hekimliği Fakültesi

Protetik Diş Tedavisi Anabilim Dalı

GSM: 05304152496

e-mail: rukiye_durkan@hotmail.com 